\title{
Respiratory effects of lowering tar and nicotine levels of cigarettes smoked by young male middle tar smokers.
}

\section{Results of a randomised controlled trial}

\author{
C H Withey, A O Papacosta, A V Swan, B A Fitzsimons, G A Ellard, P G J Burney, \\ J R T Colley, W W Holland
}

\begin{abstract}
Study objective-The aim was to investigate the effect on respiratory health of male middle tar smokers changing the tar and nicotine levels of the cigarettes they smoke for a six month period.

Design-This was a randomised controlled trial. Middle tar smokers were randomly allocated to smoke one of three different types of cigarette (low tar, middle nicotine; middle tar, middle nicotine; and low tar, low nicotine) in place of their usual cigarette for a six month period. Main outcome measures were assessment of respiratory health by documenting respiratory symptoms and peak expiratory flow rates, and of nicotine inhalation by measuring the urinary excretion of nicotine metabolites.

Setting-21 local authority districts of England.

Subjects-Participants were male middle tar smokers aged 18-44 years.

Main results-Changes in the measures of respiratory health showed little difference over the trial period between the three cigarette groups. Analyses of the urinary nicotine metabolites showed that smokers allocated to each of the three study cigarettes adjusted their smoking so that throughout the trial their nicotine inhalation differed little from their pretrial intakes when they were smoking their own cigarettes. As a result of the altered patterns of smoking to compensate for the reduced nicotine yields of the three study cigarettes, the tar intake of those allocated to smoke the middle tar, middle nicotine cigarettes remained essentially unchanged, while those allocated to smoke the low tar, low nicotine and low tar, middle nicotine cigarettes had calculated reductions in tar intakes of about $14 \%$ and $18 \%$, respectively.

Conclusions-Due to the phenomenon of compensation, tar intake can only be reduced substantially by using a cigarette with a markedly lower $\operatorname{tar} /$ nicotine ratio. Nevertheless reductions of up to about $18 \%$ in tar intake failed to result in any detectable effect on respiratory symptoms or peak expiratory flow rates over a six month period.
\end{abstract}

Tar is probably the most lethal of the harmful components of smoke and is generally held to be responsible for cigarette induced lung cancer and

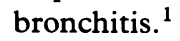

There is a generally held belief that smoking low yield cigarettes is "safer" than smoking middle or high tar brands. However, various studies have shown that when smokers change to smoking cigarettes with reduced tar and nicotine yields, they "compensate" to maintain their customary nicotine intake. ${ }^{2-10}$ This compensation can take the form of an altered pattern of smoking achieved by, for example, increasing the number of cigarettes smoked, inhaling more frequently or deeply, taking puffs of larger volume, or smoking the cigarette to shorter butt lengths. ${ }^{2}{ }^{3}$ It has been suggested that, due to compensation, firstly smokers do not consume less nicotine ${ }^{4}$; secondly, the assumption that changing to low tar cigarettes significantly reduces the risk of developing smoking related diseases may be unwarranted ${ }^{4}$; and finally, cigarettes with low nicotine yield may not be less harmful for heavy smokers. ${ }^{5}$

Since the tar/nicotine ratios of the great majority of cigarette brands are very similar, ${ }^{11}$ it was therefore apparent that substantially reduced tar intakes could only be achieved in the face of compensation if cigarettes with low tar but middle nicotine yields could be developed, as originally suggested by Russell. ${ }^{12}$ Such types of cigarettes have recently been manufactured and marketed. Therefore, both to assess changes in respiratory health and to examine this aspect of compensation, three types of cigarette were included in the main study - a low tar, middle nicotine (LM), a middle tar, middle nicotine (MM), and a low tar, low nicotine (LL) cigarette.

The hypotheses being tested in this study were that smoking lower tar cigarettes would result in fewer respiratory symptoms than continuing to smoke middle tar cigarettes, that middle tar smokers who change to low tar, low nicotine cigarettes will compensate, while those who change to low tar, middle nicotine cigarettes will not compensate and will have fewer respiratory symptoms.

\section{Methods}

The methodology of this study has been described in detail in the preceding paper. ${ }^{13}$

\section{STATISTICAL ANALYSES}

Respiratory health measures

The trial yielded repeated measurements for scores obtained from the cough frequency, as measured by the Field's card system, as well as the 
two sets of cough and phlegm statements. In the analyses of the Field's card system scores, regression coefficients (summarising the rates of change over time) were calculated for each participant in the trial excluding those with single or only two consecutive observations. The estimated rates of change in the Field's card system score over a two week period (ie, the individual regression coefficients) were then used as the outcome variable in an analysis to test for differences between the three cigarette groups allowing for independent variables such as age, compliance, cigarette consumption prior to the trial, and prevalence of respiratory symptoms at the start of the trial. Since the regression coefficients were calculated from varying numbers of observations (due to missed visits during the trial), in the analysis they were weighted using as weights the sum of squared deviations on the time axis. The magnitude of the weight thus depended not only on the number of observations used to estimate the regression coefficient but also on how widely the observations were spread on the time scale. Interactions between group and the other independent variables were tested to assess whether the effects of changing cigarette type on symptoms were affected by other independent variables. The same approach was used for the analysis of the cough and phlegm statements.

The peak expiratory flow rates were measured on two occasions, at the recruitment stage and at the 24th week of the trial. The change in the mean between the first and second occasion was used as the outcome measure for the analysis. Differences between the three cigarette groups were tested for, allowing for the same independent variables as in the analysis of the respiratory symptoms.

\section{Results}

The 265016 postal questionnaires yielded 16580 men aged 18-44 years who reported smoking only manufactured cigarettes, of whom 7736 smoked middle tar cigarettes. Of these middle tar cigarette smokers, 5153 were eventually interviewed and 2666 of these were eligible and agreed to take part in the trial.

Although 2666 individuals initially agreed at recruitment to take part in the trial, at the first visit $1125(42 \%)$ withdrew from the trial before smoking any of the trial cigarettes; $1541(58 \%)$ participants therefore began smoking the trial cigarettes, with 517,506 , and 518 in the $\mathbf{L M}$, MM, and LL groups respectively. Of these 1541 participants, $643(42 \%)$ completed the trial

Table I Weighted means (SE) of the regression coefficients summarising the rate of change per fortnightly visit for the Field's card system (FCS) and phlegm and cough scores for those initially with and without symptoms within each of the three cigarette groups

\begin{tabular}{|c|c|c|c|c|c|c|}
\hline & \multicolumn{3}{|c|}{ Without symptoms } & \multicolumn{3}{|c|}{ With symptoms } \\
\hline & $L M$ & $M M$ & $L L$ & $L M$ & $M M$ & $L L$ \\
\hline $\begin{array}{l}\text { FCS } \\
n\end{array}$ & $\begin{array}{l}0.0078 \\
(0.0133) \\
162\end{array}$ & $\begin{array}{c}-0.0035 \\
(0.0130) \\
171\end{array}$ & $\begin{array}{c}0.0429 \\
(0.0135) \\
161\end{array}$ & $\begin{array}{c}-0.0098 \\
(0.0152) \\
124\end{array}$ & $\begin{array}{c}-0.0010 \\
(0.0147) \\
141\end{array}$ & $\begin{array}{c}-0.0062 \\
(0.0149) \\
133\end{array}$ \\
\hline $\begin{array}{l}\text { Phlegm } \\
\text { n }\end{array}$ & $\begin{array}{l}0.0029 \\
(0.0055) \\
166\end{array}$ & $\begin{array}{c}0.0023 \\
(0.0053) \\
188\end{array}$ & $\begin{array}{l}0.0165 \\
(0.0055) \\
162\end{array}$ & $\begin{array}{l}0.0046 \\
(0.0074) \\
127\end{array}$ & $\begin{array}{c}-0.0111 \\
(0.0071) \\
146\end{array}$ & $\begin{array}{c}0.0027 \\
(0.0073) \\
134\end{array}$ \\
\hline $\begin{array}{l}\text { Cough } \\
\mathrm{n}\end{array}$ & $\begin{array}{l}0.0006 \\
(0.0069) \\
166\end{array}$ & $\begin{array}{l}0.0009 \\
(0.0067) \\
180\end{array}$ & $\begin{array}{c}0.0133 \\
(0.0069) \\
162\end{array}$ & $\begin{array}{c}0.0002 \\
(0.0083) \\
127\end{array}$ & $\begin{array}{c}-0.0094 \\
(0.0080) \\
146\end{array}$ & $\begin{array}{c}0.0014 \\
(0.0082) \\
134\end{array}$ \\
\hline
\end{tabular}

$\mathrm{LM}=$ low tar, middle nicotine; $\mathrm{MM}=$ middle tar, middle nicotine; $\mathrm{LL}=$ low tar, low nicotine smoking the study cigarettes, with 213,220 , and 210 in the LM, MM, and LL groups respectively.

Data on those men who agreed to participate in the trial and those who refused are given in the preceding paper. ${ }^{13}$ Results of the respiratory health measures and urine analyses will be given here.

\section{RESPIRATORY HEALTH MEASURES}

\section{Field's card system}

Table I shows the rates of change for the cough frequency measured by the Field's card system, together with those for the phlegm and cough scores within each of the three cigarette groups for all participants who were visited at least three times. The figures are given separately for those with and without symptoms before the trial, when their mean Field's card system scores were 4.2 and 2.4 respectively. Among those without symptoms, only one group (LL) had a significantly non-zero rate of change. This group showed an increase in cough frequency (Field's score) approximately equivalent to half a unit in the six month trial period. Among those with symptoms at the start of the trial all the changes were negative, that is showing a decrease in cough, but they were not significantly different from zero or each other. It was possible that other factors could have confounded the effects of the cigarettes. However, despite using weighted multiple regression and allowing for the independent effects of age, respiratory symptoms and daily cigarette consumption prior to the trial, there were still no significant differences in the mean rates of change between the three cigarette groups. Neither the effects of the independent variables nor their interactions were found to be significant.

Phlegm and cough statements

Table I shows the mean rates of change in the phlegm and cough scores. In neither case did the rates differ significantly from each other within the symptom categories. Again, the effects of age, cigarette consumption, and symptom status were taken into account in a regression analysis. Although there was evidence of an interaction between age and cigarette group in the case of cough, no other interaction or direct effects were significantly different from zero. For both phlegm and cough there was no evidence of the rates of change differing between the cigarette groups.

\section{Peak expiratory flow rate}

The peak expiratory flow rate measured at the recruitment stage averaged about 545 litres per minute. All groups showed an increase over the trial period and that for the LL group was significantly greater than zero. The analyses of the changes after allowing for the effects of age, presence of respiratory symptoms, and cigarette consumption prior to the trial showed no significant differences between the three cigarette groups.

\section{COMPLIANCE}

Of the cigarette butts collected at visits 3 and 13, $91 \%$ were from the allocated study cigarettes with very similar numbers in each of the three cigarette groups. This shows that significant bias was not introduced into the study by participants smoking non-study cigarettes. 
INHALATION OF NICOTINE AND CIGARETTE CONSUMPTION

Urinary nicotine metabolite/creatinine ratios for the 296 subjects from whom urine samples and cigarette consumption reports were obtained on each of five scheduled visits were calculated and are summarised in table II. These have been corrected by subtracting a value of 0.8 to allow for the contribution to the ratio from normal components in urine samples from non-smokers. ${ }^{14} \mathrm{~A}$ comparison of expected and actual changes in urinary nicotine metabolite/creatinine $(\mathrm{NM} / \mathrm{C})$ ratios, representing nicotine intake, for the subset of individuals providing urine specimens is given in table III. The expected changes in urinary nicotine metabolite/creatinine ratio, that is the changes that would have been expected if the participants had smoked the trial cigarettes in exactly the same way as their own pretrial brands, were obtained by calculating what nicotine metabolite/creatinine ratio would be expected for the given changes in nicotine levels and consumption and subtracting this from the pretrial values. Since the actual changes in nicotine metabolite/ creatinine ratios estimated to have occurred were not significantly different from zero in any of the three groups it appears that the participants had modified their pattern of smoking so that throughout the trial their nicotine inhalation differed little from their pretrial intakes when they were smoking their own cigarettes. This was despite reductions in nicotine yields of between 13 and $27 \%$ and an increase in reported daily cigarette consumption of about $5 \%$.

Similar compensation was shown in the feasibility study when men were switched to a low tar,

Table II Urinary excretion of total nicotin metabolites by allocated trial cigarette group for the 296 subjects from whom urine samples and cigarette consumption reports were obtained on each of the five scheduled visits

\begin{tabular}{|c|c|c|c|}
\hline \multirow{2}{*}{$\begin{array}{l}\text { Week of } \\
\text { trial }\end{array}$} & \multicolumn{3}{|c|}{ Cigarette group } \\
\hline & $L M$ & $M M$ & $L L$ \\
\hline $\begin{array}{l}0 \\
\text { (own cigarettes) } \\
2 \\
4 \\
12 \\
24 \\
\text { Mean of weeks } 2-24\end{array}$ & $\begin{array}{l}10.9(0.6)^{\mathrm{a}} \\
11.3(0 \cdot 6) \\
11.5(0 \cdot 6) \\
11.8(0 \cdot 7) \\
12.1(0 \cdot 7) \\
11.7(0 \cdot 3)\end{array}$ & $\begin{array}{l}10.8(0.6) \\
10.7(0.6) \\
10.5(0.6) \\
10.5(0.5) \\
10.5(0.5) \\
10.5(0.3)\end{array}$ & $\begin{array}{l}11.4(0.6) \\
11.2(0.6) \\
11.1(0.6) \\
11.9(0.6) \\
11.1(0.5) \\
11.3(0.3)\end{array}$ \\
\hline
\end{tabular}

${ }^{a}$ Mean $(\mathrm{SE}) \mu \mathrm{g}$ total nicotine metabolites per $\mathrm{mg}$ creatinine $\mathrm{LM}=$ low tar, middle nicotine; $\mathrm{MM}=$ middle tar, middle nicotine; $\mathrm{LL}=$ low tar, low nicotine

Table III Observed and expected changes in nicotine intake for the 296 subjects from whom urine samples and cigarette consumption reports were obtained on each of the five scheduled visits

\begin{tabular}{|c|c|c|c|c|}
\hline & \multicolumn{3}{|c|}{ Trial cigarette group } & \multirow{2}{*}{$\begin{array}{l}\text { Pretrial } \\
\text { cigarette }\end{array}$} \\
\hline & $\overline{L M}$ & $M M$ & $L L$ & \\
\hline $\begin{array}{l}\text { Tar yield (mg/cigarette) } \\
\text { Nicotine yield (mg/cigarette) } \\
\text { Tar/nicotine ratio }\end{array}$ & $\begin{array}{l}9 \cdot 5 \\
1 \cdot 16 \\
8 \cdot 2\end{array}$ & $\begin{array}{l}13 \cdot 8 \\
1 \cdot 24 \\
11 \cdot 1\end{array}$ & $\begin{array}{l}9 \cdot 7 \\
1 \cdot 04 \\
9 \cdot 3\end{array}$ & $\begin{array}{l}15 \cdot 3 \\
1 \cdot 43 \\
10 \cdot 7\end{array}$ \\
\hline $\begin{array}{l}\text { Mean } \% \text { fall in nicotine yield due to } \\
\text { switching to trial cigarette }\end{array}$ & 19 & 13 & 27 & \\
\hline $\begin{array}{l}\text { Mean pretrial } N M / C \text { ratio } \\
\text { Mean trial } N M / C \text { ratio }\end{array}$ & $\begin{array}{l}10 \cdot 9 \\
11 \cdot 7\end{array}$ & $\begin{array}{l}10 \cdot 8 \\
10 \cdot 5\end{array}$ & $\begin{array}{l}11 \cdot 4 \\
11 \cdot 3\end{array}$ & \\
\hline \multirow{2}{*}{$\begin{array}{l}\text { Mean pretrial cigarette consumption } \\
\text { Mean trial cigarette consumption } \\
\% \text { Increase in cigarette consumption from } \\
\text { pretrial to trial }\end{array}$} & $\begin{array}{l}19 \cdot 2 \\
20 \cdot 0\end{array}$ & $\begin{array}{l}17 \cdot 8 \\
18 \cdot 7\end{array}$ & $\begin{array}{l}19 \cdot 0 \\
19 \cdot 9\end{array}$ & \\
\hline & $4 \cdot 2$ & $5 \cdot 1$ & $4 \cdot 7$ & \\
\hline $\begin{array}{l}\text { Expected drop in NM/C ratio } \\
\text { Observed drop in NM/C ratio between } \\
\text { visit } 1 \text { and mean of visits } 2,3,7 \text {, and } 13 \text { of } \\
\text { the trial (SE) } \\
\text { Difference }\end{array}$ & $\begin{array}{l}-0 \cdot 8(0 \cdot 6) \\
2 \cdot 5\end{array}$ & $\begin{array}{l}0.3(0.4) \\
0.7\end{array}$ & $\begin{array}{l}0 \cdot 1(0 \cdot 4) \\
2 \cdot 6\end{array}$ & \\
\hline $\begin{array}{l}\% \text { Reduction in tar intake from pretrial to } \\
\text { trial }\end{array}$ & 18 & 0 & 14 & \\
\hline
\end{tabular}

low nicotine cigarette. ${ }^{11}$ Armitage et al ${ }^{15}$ have recently shown a similar phenomenon using steady state cotinine estimations in a crossover study conducted among a group of 21 smokers who had been randomly allocated to smoke middle tar, middle nicotine cigarettes and a "maintained nicotine" brand of cigarette, with tar and nicotine yields similar to those of our LM cigarettes.

The tar yields of the brands smoked by the participants at entry to the study averaged $15 \cdot 3$ $\mathrm{mg} /$ cigarette, giving a mean tar/nicotine ratio of 10.7. By contrast the tar yields of the three trial cigarettes were $9.5,13.8$, and $9.7 \mathrm{mg} /$ cigarette, giving $\operatorname{tar} /$ nicotine ratios of $8 \cdot 2,11 \cdot 1$, and $9 \cdot 3$ for the LM, MM, and LL cigarettes, respectively. Since both nicotine and tar are found in the particulate fraction of cigarette smoke, likely changes in tar intake could be calculated from the relative amounts of nicotine inhaled when the smokers were smoking their own cigarettes and when they smoked the three trial cigarettes, as indicated by urinary nicotine metabolite/ creatinine ratios (table II), and the tar/nicotine ratios of the trial and pretrial cigarettes. The nicotine intake of the men allocated to smoke the LM cigarettes increased by $7^{\circ}$ (nicotine metabolite/creatinine ratios from 10.9 to 11.7 , table II) but because the tar/nicotine ratios for their cigarettes fell by $23^{\circ}$ o (10.7 to 8.2 ), this gave a net estimated reduction in tar intake of $18^{\circ}{ }_{0}$. Similar calculations indicated a reduction of about $14 \%$ in those allocated to smoke the LL cigarettes, while the tar intake of those who smoked the MM cigarettes was essentially unchanged.

\section{Discussion}

A smoker's choice of cigarette may be affected by his existing symptoms, which means that an observational study comparing high and low tar smokers may be considerably affected by selection bias. It was therefore considered essential to conduct an intervention study where smokers were randomly allocated to different types of cigarette. During the study it was observed that there was some evidence that individuals were more likely to agree to participate in the trial if they had higher cigarette consumption levels or had respiratory symptoms. ${ }^{13}$ However the randomised trial design ensured that the individuals in the three cigarette groups would, apart from predictable sampling error, be comparable.

The lowering of tar consumption was to be achieved by participants smoking trial cigarettes with tar levels lower than those of their usual cigarettes. However, because tar and nicotine levels in tobacco are positively correlated, practically all participants were changed to trial cigarettes which also had lower nicotine levels than their usual brand. This meant that to obtain the same level of nicotine intake as before they would have to smoke more cigarettes or smoke them more intensively. It is known that smokers may alter their pattern of smoking when changing to lower tar, lower nicotine cigarettes and because such compensation would offset the reduction in tar consumption, it was important to assess to what extent it occurred in each of the three cigarette groups. 
Nicotine is a powerful addictive substance and has an important psychophysiological effect on smoking behaviour. It is therefore not really feasible to lower the nicotine yield of cigarettes beyond the minimum requirements of smokers. If it is the reduced nicotine intake that causes a smoker to compensate when changing to a $\mathrm{LL}$ cigarette, this should mean that less compensation would occur when changing to a LM cigarette with a corresponding reduction in the amount of tar inhaled. Therefore reducing other harmful constituents of smoke such as tar should be acceptable to smokers as long as they can maintain the same nicotine intake. However, the machine yields can be misleading, as people do not smoke the same way as machines-cigarette smokers smoke in such a way as to optimise the intake of nicotine. ${ }^{16}$ Cigarette smokers are also able to regulate their smoking behaviour so as to maintain their body nicotine concentration within close limits. ${ }^{17}$ As a result of this human compensatory smoking, the reduction in tar and nicotine may be less than expected when changing to a lower tar, lower nicotine cigarette. The mean levels of tar and nicotine in the cigarettes smoked by all participants prior to the study were $15.3 \mathrm{mg}$ and $1.4 \mathrm{mg}$ per cigarette respectively. The LM group changed to approximately $9.5 \mathrm{mg}$ tar and $1.16 \mathrm{mg}$ nicotine per cigarette; the MM group to $13.8 \mathrm{mg}$ tar and $1.24 \mathrm{mg}$ nicotine; and the LL group to $9.7 \mathrm{mg}$ tar and $1.04 \mathrm{mg}$ nicotine. Thus all subjects experienced a drop in both the nicotine and the tar level of their cigarettes.

The outcome analyses focused on the respiratory symptoms observed throughout the trial, namely cough frequency measured by Field's card system and the statements on cough and phlegm and a measure of lung function, the peak expiratory flow rate. The results showed that no systematic patterns of change occurred in any group although there was a slight, but generally non-significant, tendency for the "with symptoms" group to improve and the "without symptoms" group to get worse. This could be the result of some regression to the mean. The analyses to compare the patterns of change in the symptom measures used the "within subjects" rates of change as the outcome variable. This allowed the analyses to include individuals from whom data were not obtained for all of the visits. The mean rates of change for Field's card system score were all negative in those "with symptoms" with two out of three positive in those "without symptoms". However, only in one case, the "without symptoms" LL group, did the rate of change differ significantly from zero. Thus there is little scope for the groups to differ significantly from each other even in a multiple regression analysis adjusting for age and other effects to increase sensitivity. In fact age did not appear to have any appreciable effect and there was no evidence of any difference between cigarette groups regardless of whether the analysis allowed for symptom status. Similar analyses for the five point scores for cough and phlegm gave much the same results. Thus there was no evidence of systematic differences in the rates of change between groups allocated to smoke different cigarettes.
The results for the peak expiratory flow rate showed that there was a slight increase in the mean for all three cigarette groups and the increase in the LL group reached statistical significance. There was no obvious explanation for this. After allowing for other factors the changes in peak expiratory flow rate during the trial in the three cigarette groups were not significantly different from each other.

Although there was some evidence of systematic changes in cough and phlegm frequencies and the peak expiratory flow rates during the six month trial period, significant differences in respiratory health could not be shown between the three study cigarette groups. This was despite the fact that the study size had been chosen to have a power of $90 \%$ of detecting significant biological effects. ${ }^{13}$ The variation of the within subject peak expiratory flow rate differences was such that the approximately 225 participants in each group gave a power of $90 \%$ of detecting differences in peak expiratory flow rate changes of the order of $4 \%$. This means that if the real effect of switching to the trial cigarettes was no change in peak expiratory flow rate for the MM group and a $4 \%$ increase ( 545 to 567 litres/min) for the LM group, there was a $90 \%$ chance that this study would detect it. This applies equally to comparisons of $2 \%$ and $6 \%$ increases or $10 \%$ and $14 \%$. Significant short term benefits are unlikely to be gained from reductions in tar intake of up to about $18 \%$, the reduction achieved in this study by switching to low tar, middle nicotine cigarettes.

The analysis discussed above relies on the comparison of groups which does not take into account individual variation in the levels of compensation. In the trial, individuals experienced a range of different changes in tar intake which can be estimated using the change in nicotine metabolite/creatinine ratios. A further analysis was performed assessing the relationship between changes in the measures of respiratory symptoms and these estimated individual reductions in tar consumption, but there was still no evidence of an association.

The study also showed that as a result of compensation, changing to generally available low tar cigarettes results in much smaller reductions in tar intake than might be expected on the basis of smoking machine tar yields.

As significant reductions in tar intake might in the long run still lead to reduction in the risk of smoking induced lung cancers and bronchitis, smokers who are unwilling to stop smoking should still be encouraged to switch to low tar cigarettes. Since this study has shown that substantially greater reductions in tar intake can be achieved by using a low tar, middle nicotine cigarette, the tobacco industry should be encouraged to make such brands available on the market. Nevertheless, this study shows that altering the composition of cigarette tobacco is not likely to have any detectable effect on respiratory health in a period of six months. Substantial benefits can only be obtained by persuading individuals to stop smoking entirely.

We should like to thank Dr Michael Hensley, Dr Fiona North, and Mrs Joanna Terry for their help in this study, Ms Nicky Smith for secretarial support, Ms 
Kalavati Patel for statistical assistance, and Social and Community Planning Research for undertaking the sampling and fieldwork. We should also like to thank Rothmans, Gallaher, and Imperial Tobacco Companies for manufacturing the trial cigarettes. The study was funded by the Tobacco Products Research Trust.

1 Royal College of Physicians. Smoking and health now. London: Pitman, 1971.

Sutton SR, Russell MAH, Iyer R, Feyerabend C, Saloojee

Y. Relationship between cigarette yields, puffing patterns and smoke intake. Evidence for tar compensation. $B M \mathcal{F}$ and smoke intake.

3 Ebert RV, McNabb E, McCusker K, Snow S. Amount of nicotine and carbon monoxide inhaled by smokers of nicotine and carbon monoxide inhaled by smokers of low-tar, low-

4 Benowitz NL, Hall SM, Herning RI, Peyton J, Jones RT, Osman A. Smokers of low-yield cigarettes do not consume less nicotine. $N$ Engl f Med 1983; 309: 139-42.

5 Russell MAH, Wilson C, Patel UA, Cole PV, Feyerabend C. Comparison of effect on tobacco consumption and carbon monoxide absorption of changing to high and low nicotine cigarettes. $B M \mathcal{F}^{\prime} 1973$; iv: $512-6$.

6 Turner JA, Sillett RW, Ball KP. Some effects of changing to low-tar and low-nicotine cigarettes. Lancet 1974; ii: 737-9.

7 Herning RI, Jones RT, Bachman J, Mines AH. Puff volume increases when low-nicotine cigarettes are smoked. $B M \mathcal{Y}$ 1981; 283: 187-9.
8 Petitti DB, Friedman GD. Evidence for compensation in smokers of low yield cigarettes. Int $\mathcal{f}$ Epidemiol 1983; 12: 487-9.

9 Woodman G, Newman SP, Pavia D, Clarke SW. Response and acclimatisation of symptomless smokers on changing to low tar, low nicotine cigarette. Thorax 1987; 42: 336-41. 10 Russell MAH, Sutton SR, Iyer R, Feyerabend C, Vesey CI. Long-term switching to low tar-low-nicotine cigarettes. $B r$ Long-term switching to low
$\mathcal{F}$ Addict 1982; 77: 145-58.

11 Peach H, Ellard GA, Hayward DM, Shah D. A double blind randomised controlled trial of the effect of a low versus a middle tar cigarette on respiratory symptoms-a feasibility middle tar cigarette on respiratory sympto

12 Russell MAH. Low-tar, medium-nicotine cigarettes: A new approach to safer smoking. $B M F$ 1976; i: $1430-3$.

13 Withey CH, Papacosta AO, Swan AV, et al. Respiratory effects of lowering tar and nicotine levels of cigarettes smoked by young male middle tar smokers. I. Design of a randomised controlled trial. $\mathcal{f}$ Epidemiol Community Health 1992; 46: 274-80.

14 Peach H, Ellard GA, Jenner PJ, Morris RW. A simple inexpensive urine test of smoking. Thorax 1985; 40: 351-7.

5 Armitage AK, Alexander J, Hopkins R, Ward C. Evaluation of a low to middle tar/medium nicotine cigarette designed to maintain nicotine delivery to the smoker. Psychopharmacology 1988; 96: 447-53.

6 Hughes JR, Hatsukami D. Signs and symptoms of tobacco withdrawal. Arch Gen Psychiatry 1986; 43: 289-94.

17 Benowitz NL. Pharmacologic aspects of cigarette smoking and nicotine addiction. N Eng $\mathcal{f}$ Med $1988 ; 319$ : 1318-30.

Extract from Dr Ferriar's Advice to the Labouring Classes in Manchester; given in 1800 Avoid living in damp cellars; they destroy your constitutions and shorten your lives. No temptation of low rents can counterbalance their ill effects. You are apt to crowd into the cellars of new buildings, supposing them to be clean; this is a fatal mistake; a new house is always damp for two years, and the cellars which you inhabit under them are generally as moist as the bottom of a well. In such places you are liable to bad fevers, which often throw the patient in to a decline, and you are apt to get rheumatic complaints, that continue for a long time and disable you from working.

If you cannot help taking a cellar, be attentive to have all the windows put into good repair before you venture into it, and, if possible, get it white-washed. If you attempt to live in a cellar with broken windows, colds and fevers will be the certain consequences.

In many parts of the town you sleep in back rooms, behind the front cellar, which are dark and have no proper circulation of air. It would be much more healthy to sleep to the front; at least when you have large families, which is often the case, you ought to divide them, and not to crowd the whole together in the back cellar.

Keep your persons and houses as clean as your employments will permit, and do not regret the loss of an hour's wages when your time is occupied in attending to cleanliness. It is better to give up a little time occasionally to keep your homes neat, than to see your whole family lying sick in consequence of working constantly without cleaning. It would be of great service if you could contrive to air your bed and bed-clothes out of doors once or twice a-week.

Always wash your children from head to foot with cold water before you send them to work in the morning. Take care to keep them dry in their feet, and never allow them to go to work without giving them their breakfast, though you should have nothing to offer them but a crust of bread and a little water. Children who get wet feet, when they go out early fasting, seldom escape fever or severe colds.

Your health will always be materially injured by the following circumstances:-living in small back buildings, adjoining to the open vaults of privies; living in cellars where the streets are not properly soughed or drained; living in narrow bye-streets where sheep are slaughtered, and where the blood and garbage are allowed to stagnate and corrupt, and perhaps more than all, by living crowded together in dirty lodging houses where you cannot have the common comforts of light and air.

It should be unnecessary to remind you that much sickness is occasioned among you by passing your evenings at ale-houses, or in strolling about the streets or in the fields adjoining to the town. Perhaps those who are most apt to expose themselves in this manner would pay little attention to dissuasive arguments of any kind; however, those who feel an interest in your welfare cannot omit making the remark.

Report to Her Majesty's Principal Secretary of State for the Home Department, from the Poor Law Commissioners on an inquiry into the Sanitary Condition of the labouring population of Great Britain, 1842 